(2) Open Access Full Text Article

\title{
Astaxanthin Attenuates Neuroinflammation in Status Epilepticus Rats by Regulating the ATP-P2X7R Signal
}

This article was published in the following Dove Press journal: Drug Design, Development and Therapy

\author{
Ming Wang ${ }^{1,2}$ \\ Xiaolin Deng (D) ${ }^{1,2}$ \\ Yangmei Xie ${ }^{1,2}$ \\ Yinghui Chen (1D) ${ }^{1,2}$ \\ 'Department of Neurology, Jinshan \\ Hospital, Fudan University, Shanghai, \\ People's Republic of China; ${ }^{2}$ Department \\ of Neurology, Huashan Hospital North, \\ Fudan University, Shanghai, People's \\ Republic of China
}

Background: As a life-threatening neurological emergency, status epilepticus (SE) is often refractory to available treatment. Current studies have shown a causal role of neuroinflammation in patients with lower seizure thresholds and driving seizures. The ATP-gated purinergic $\mathrm{P} 2 \mathrm{X} 7$ receptor ( $\mathrm{P} 2 \mathrm{X} 7 \mathrm{R})$ is mainly expressed on the microglia, which function as gatekeepers of inflammation. Although emerging evidence has demonstrated significant anti-inflammatory effects of astaxanthin (AST) in SE, the associated mechanism remains unclear. Therefore, this study aimed to clarify the effects of AST on P2X7R-related inflammation in SE.

Methods: SE was induced in rats using lithium-pilocarpine, and AST was administered 1 $\mathrm{h}$ after SE induction. Rat microglia were treated with lipopolysaccharide (LPS), AST, ATP, 2,3-O-4-benzoyl-4-benzoyl-ATP (BzATP) and oxidized ATP (oxATP). The Morris water maze, immunohistochemistry, and Nissl staining were performed in rats. Expressions of P2X7R and inflammatory cytokines (such as cycloxygenase-2 (Cox-2), interleukin-1 $\beta$ (IL$1 \beta)$, and tumor necrosis factor- $\alpha(\mathrm{TNF}-\alpha)$ ) were detected using real-time polymerase chain reaction (RT-PCR) and Western blot (WB) both in rats and microglia. ATP concentration in the microglia was evaluated using ELISA.

Results: The AST alleviated hippocampal injury and improved cognitive dysfunction induced by SE. AST also effectively inhibited inflammation and downregulated P2X7R expression in both rat brain and microglia. The results also showed that AST reduced the extracellular ATP levels and that P2X7R expression could be increased by extracellular ATP. In addition, BzATP upregulates the expression of $\mathrm{P} 2 \mathrm{X} 7 \mathrm{R}$ and inflammatory factors in microglia. Conversely, it downregulates the expression of P2X7R and inflammatory factors. Conclusion: Our study suggests that AST attenuated ATP-P2X7R mediated inflammation in SE.

Keywords: status epilepticus, astaxanthin, neuroinflammation, P2X7R, ATP

\section{Introduction}

As a common and severe neurological emergency condition, status epilepticus (SE) is defined as follows: continuous or recurrent seizures lasting for minimum of 5 mins, along with an incomplete recovery of consciousness between seizures. ${ }^{1-3}$ The incidence of SE is approximately 61 out of every 100,000 people per year, with long-term sequelae and high mortality rates. ${ }^{1}$ The etiology of SE is particularly complex, including structural causes, central nervous system infections, irregular use of anti-epileptic drugs, and rare autoimmune causes. ${ }^{2}$ Current treatment modalities are mainly based on
Correspondence: Yinghui Chen Department of Neurology, Huashan Hospital North, Fudan University, Shanghai, People's Republic of China Tel +86 I8930819605

Email yinghuichen@fudan.edu.cn 
expert consensuses, since anti-epileptic drugs do not appear to significantly influence the disease prognosis. Therefore, a better understanding of the underlying mechanisms of SE is urgently needed, which may subsequently contribute to the development of new therapeutic strategies. Increasing evidence from patients and animal models of SE demonstrated that inflammation is a facilitator and a consequence of seizures. ${ }^{4}$ Microglia are immune effector cells inherent in the central nervous system, which can be rapidly activated in response to various stimuli. ${ }^{5,6}$ The ATP-gated purinergic $\mathrm{P} 2 \mathrm{X} 7$ receptor $(\mathrm{P} 2 \mathrm{X} 7 \mathrm{R})$ is an ion channel receptor located on the surface of microglia and has been previously reported. $^{7,8} \mathrm{P} 2 \mathrm{X} 7 \mathrm{R}$ can be activated by the ATP efflux when the microglia exposure to noxious stimuli such as seizures, stroke, and trauma, ${ }^{9}$ followed by activation of the P2X7R-mediated NLRP3 inflammasome and the subsequent release of inflammatory cytokines. ${ }^{10}$ Additionally, microglia were further activated by self-released inflammatory factors, which further aggravate the inflammatory cascade. Therefore, P2X7R is considered the gatekeeper of inflammation. Consistently, current evidences demonstrate that P2X7R expression was dysregulated after SE in both experimental animals and patients. ${ }^{11}$ In addition, emerging studies have focused on the development of P2X7R antagonists as novel anti-epileptic drugs. ${ }^{12}$ Therefore, P2X7R play an important role in SE-induced inflammation.

Astaxanthin (AST) belongs to the carotenoid family and has strong anti-inflammatory and antioxidant effects. Natural AST is mainly derived from algae, yeast, and marine life such as crab, fish, and shrimp. ${ }^{13}$ In the past two decades, several researchers have sought to determine the specific mechanism underlying the anti-inflammatory and anti-oxidation effects of AST. Most studies have reported that AST inhibits reactive oxygen species production, maintains the structural integrity of mitochondria, suppresses the inflammatory signaling pathway, and attenuates cellular dysfunction. ${ }^{14-16}$ Similarly, a recent study indicated that AST reverses the damage to hippocampal neurons in neonatal rats exposed to maternal seizure, which may contribute to the reduction of oxidative stress. ${ }^{17}$ Moreover, increasing evidence has demonstrated that AST inhibits ATP depletion to maintain the energy supply to the cells. ${ }^{13,14}$ Our previous studies also suggested that AST attenuated SE-induced cognitive dysfunction through inhibited oxidative stress, neuroinflammation, and neuronal apoptosis in rat brains. ${ }^{18}$ However, there are no studies have investigated whether AST influences neuroinflammation by regulating the ATP-P2X7R signal in
SE. Therefore, in this study, we aimed to confirm whether AST regulates the expression of $\mathrm{P} 2 \mathrm{X} 7 \mathrm{R}$ and to explore its new potential anti-inflammatory mechanisms both in vivo and vitro.

\section{Materials and Methods \\ Reagents}

Lithium chloride, pilocarpine, polyethylene glycol, lipopolysaccharide (LPS), ATP, 2,3-O-4-benzoyl-4-benzoyl-ATP (BzATP) and oxidized ATP (oxATP) were purchased from Sigma (USA). Diazepam was obtained from Yimin (Beijing, China), AST from Solarbio (Beijing, China), dimethyl sulfoxide (DMSO) from Amresco (Beijing, China), fetal bovine serum (FBS), Dulbecco's modified eagle medium (DMEM) high glucose medium from Gibco (USA), and the trizol reagent and RT-PCR kits from Takara (Shanghai, China). P2X7R polyclonal antibody\#A10511, TNF- $\alpha$ polyclonal antibody\#A11534, IL-1 $\beta$ polyclonal antobody\#A16288, Iba1 polyclonal antibody, and anti- $\beta$-actin antibody\#AC026 were purchased from ABclonal (Wuhan, China). Cox-2 (D5H5) ZP ${ }^{@}$ Rabbit mAb\#12282 was obtained from Cell Signaling Technology (USA). HRP-conjugated Affinipure Goat Anti-Rabbit IgG (H+L) \#SA00001-2 was obtained from Proteintech (Shanghai, China). ATP enzyme-linked immunosorbent assay (ELISA) kits were obtained from Halin company (Shanghai, China), and Nissl stain kits and immunohistochemical stain kits were obtained from Ruibo company (Shanghai, China).

\section{Animal Model of Status Epilepticus}

All animal experiments were performed in accordance with animal institution guidelines. Male Wistar rats (160-$180 \mathrm{~g}$ ) were purchased from the Animal Center of Fudan University (Shanghai, China). The rats were housed in standard animal housing. Rats were housed at a controlled temperature $\left(21-25^{\circ} \mathrm{C}\right)$ and $12 \mathrm{~h}$ cycles of light and dark; they were free to move and feed in the cage. One week after the rats were reared, an SE model was established using lithium chloride and pilocarpine according to the protocols mentioned in a previous study. ${ }^{19,20}$ Twenty-four hours before pilocarpine injection, the rats were intraperitoneally injected with lithium chloride $(127.3 \mathrm{mg} / \mathrm{kg}$ in $0.9 \%$ saline $)$. Thirty minutes before pilocarpine injection, scopolamine $(1 \mathrm{mg} / \mathrm{kg}$ in $0.9 \%$ saline) was intraperitoneally injected to reduce peripheral side effects. SE and AST groups were intraperitoneally 
injected with $30 \mathrm{mg} / \mathrm{kg}$ pilocarpine, and normal saline was injected into the control group, as described in our previous study described. ${ }^{18}$ Behavioral seizures were scored using the modified Racine scale. ${ }^{21}$ Seizures were graded using the following classifications: I-motionless or wet dog shaking; II-rhythmically nodding and chewing; IIIunilateral forelimb clonicity seizures; IV-bilateral forelimb clonic and convulsive standing seizures; V-withdrawal, tumbling, and tonic seizures. When the rats reached a seizure score of IV-V within $30 \mathrm{~min}$ and demonstrated a sustained state for one hour, the SE model was considered successfully introduced. If the rats had seizures that did not reach grade $I V-V$, the rats were injected with $10 \mathrm{mg} / \mathrm{kg}$ pilocarpine every $30 \mathrm{~min}$ until the seizures reached grade IV-V. No rats received pilocarpine in excess of $60 \mathrm{mg} / \mathrm{kg}$. Diazepam $(10 \mathrm{mg} / \mathrm{kg})$ was administered after $60 \mathrm{~min}$ to stop the seizures. Fifty rats were used in our study. The control group (10 rats) was injected with normal saline, and 40 rats were injected with pilocarpine to induce SE. Twenty-eight rats were successfully induced (success rate $70 \%$ ). SE could not be induced in seven rats, and five rats died (mortality rate $12.5 \%$ ). These rats were randomly divided into the SE group (14 rats) and the AST group (14 rats).

\section{Animal Experiment Protocol}

After one hour of the onset of SE, AST $(30 \mathrm{mg} / \mathrm{kg}$, dissolved in 1) was intraperitoneally injected in the AST group, as previously described. The control group and the SE group were injected with an equal amount of $0.9 \%$ saline. AST was continuously injected for 2 weeks and was administered every other day in the AST group. During the period of 2 weeks, the rats in the AST-treated group demonstrated a lower frequency of seizure occurrence than the SE group. Two weeks later, four rats from the SE group (mortality rate $28.6 \%$ ) and two from the AST group (mortality rate $14 \%$ ) died. The rats were sacrificed using an intraperitoneal injection of $10 \%$ chloral hydrate according to the Chinese Medical Sciences Animal Experiment Guide.

\section{Morris Water Maze Test}

The Morris water maze test includes a circular pool and monitoring equipment, as described in a previous study. ${ }^{22}$ The pool has a hidden platform where rats can swim to reach and escape from the water. The 14th day after the SE model was established, rats were trained to swim ( $\mathrm{n}=8$ each group), and the test was initiated on the 15th day. Warm water (25 \pm
$1{ }^{\circ} \mathrm{C}$ ) was subsequently added to the pool. The hidden platform test lasted for a period of 4 days and the rats were trained 4 times a day. In the experiment, rats were placed in four quadrants of the circular pool. The time required for the rats to find the underwater platform and stand on it was recorded as the escape latency. Rats were removed from the pool after standing on the platform for $15 \mathrm{~s}$. If the rats could not find the platform within $60 \mathrm{~s}$ after entering the water, they were gently placed on the platform for $15 \mathrm{~s}$, followed by removing them from the pool. For the spatial probe test, the platform was removed, and the rats were placed in the same entrance as before. The swimming path was recorded for $60 \mathrm{~s}$, and the time spent in the target quadrant was used to assess the spatial positioning ability.

\section{Nissl Stain}

The rats in each group were sacrificed according to the Chinese Medical Sciences Animal Experiment Guide. The brain tissues of rats were removed and placed on ice. The fresh rat brain tissues were fixed in $4 \%$ formalin solution and dehydrate, which were then sliced into $5 \mu \mathrm{m}$ thick sections and placed in cresyl violet stain ( $1 \mathrm{~g}$ cresyl violet dissolved in $100 \mathrm{~mL}$ of distilled water). This was followed by placing the dyeing tank in an incubator $\left(56^{\circ} \mathrm{C}\right)$ for $1 \mathrm{~h}$ and heating with an alcohol lamp (10 min). The sections were then rinsed with distilled water and placed into the Nissl stain; differentiation was allowed for 1 to $3 \mathrm{~min}$ and was observed under a microscope until the background was nearly colorless. The sections were then dehydrated rapidly with anhydrous ethanol and sealed with a neutral gum. The pyramidal neurons in the hippocampus were observed under a microscope within a fixed area (400× magnification) of the CA1 and CA3, as described in previous studies. ${ }^{23}$ The cell density per square millimeter and the layer thickness in the CA1 and CA3 areas of the dorsal lateral hippocampus were measured using the slices at $400 \times$ magnification. The photographs were processed by ImageJ software (USA), which counted the number of stained hippocampal integral neurons per $0.1 \mathrm{~m} 2$ of CA1 and CA 3 regions of the hippocampus. The average value was calculated for the number of positive cells: 6 rats per group, one slice per rat per region. ${ }^{24}$

\section{Immunohistochemistry}

Immunohistochemical analysis was performed by following the kit instructions. The fresh brain tissue sections ( 5 $\mu \mathrm{m})$ were heated at $60{ }^{\circ} \mathrm{C}$, followed by dewaxing and permeabilizing. The sections were then incubated with $3 \% \mathrm{H}_{2} \mathrm{O}_{2}$ for $10 \mathrm{~min}$ at room temperature. The sections 
were then washed thrice with PBS, followed by addition of goat serum for 20 min to block non-specific antigen binding. Rabbit anti-Ibal primary antibody was added and incubated overnight at $4{ }^{\circ} \mathrm{C}$. After washing with PBS for 3 times, the tissue sections were incubated with goat anti-rabbit $\operatorname{IgG}(\mathrm{H}+\mathrm{L})$ for $60 \mathrm{~min}$. Tissue sections were washed 3 times in PBS for 5 min each time, and a chromogenic substrate was added, followed by rinsing with tap water. After hematoxylin staining, the sections were rinsed with $0.1 \% \mathrm{HCl}$-ethanol, then dehydrated in graded ethanol and fixed in resin. They were then magnified 400 times under a microscope to identify and select the $\mathrm{CA} 1$ and $\mathrm{CA} 3$ regions of the hippocampus to observe the number of microglia, as described in previous studies. ${ }^{23}$ The images were processed by ImageJ software (USA), which was used to count the number of Iba1 stained microglia per $0.1 \mathrm{~m} 2$ of $\mathrm{CA} 1$ and $\mathrm{CA} 3$ regions of the hippocampus. The average value was calculated for the number of positive cells: 6 rats per group, one slice per rat per region. ${ }^{25}$

\section{Cell Culture}

Rat microglia was obtained from ScienCell (USA) and were cultured in DMEM, a high glucose medium with $10 \%$ FBS. The rat microglia were incubated in a humid mixed atmosphere of $5 \% \mathrm{CO} 2$ and $95 \%$ air at $37{ }^{\circ} \mathrm{C}$. When the cell coverage in the culture dish reached $65 \%$, it was treated with different reagents (for example, LPS $10 \mu \mathrm{g}$ / $\mathrm{mL}$, AST $50 \mu \mathrm{M}$, BzATP $150 \mu \mathrm{M}$, and oxATP $150 \mu \mathrm{M}$ ) according to the cell group. RNA and protein were extracted 48 hours after treatment with the reagent.

\section{Enzyme-Linked Immunosorbent Assay (ELISA)}

Rat microglia were treated with LPS $(10 \mu \mathrm{g} / \mathrm{mL})$ and AST for $48 \mathrm{~h}$. The culture medium was harvested, and the rat microglia were treated with the RIPA Lysis Buffer (Shanghai, China) for $15 \mathrm{~min}$, centrifuged at $14,000 \mathrm{rpm}$ for $15 \mathrm{~min}$, and the supernatant was collected. The ATP concentration was measured via an ELISA kit according to the instructions described in a previous study. ${ }^{26}$ Spectrophotometric value was measured at $450 \mathrm{~nm}$ using a microplate reader (SpectraMax, Molecular devices, USA).

\section{Western Blot (WB)}

Rat brain tissues (hippocampus and cortex) and microglia cell proteins were extracted using 1\% PMSF (phenylsulfonyl;
Beyotime, Shanghai, China) as previously described. ${ }^{27}$ The protein $(15 \mu \mathrm{g})$ was separated by using SDS-PAGE electrophoresis and transferred to a PVDF membrane. The PVDF membrane was blocked with 5\% skim milk for 1 hour at room temperature. The membrane was then washed thrice with TBST for 15 mins each time and incubated with the primary antibody (P2X7R antibody, Cox-2 antibody, IL-1 $\beta$ antibody and TNF- $\alpha$ antibody) overnight at $4{ }^{\circ} \mathrm{C}$. The membrane was washed with TBST and incubated with HRP-conjugated Affinipure goat anti-rabbit $\operatorname{IgG}(\mathrm{H}+\mathrm{L})$ for 1 hour at room temperature. The signals of the Western blots were detected by the ECL-Plus kit (Merck Millipore, Darmstadt, Germany) and quantified use ImageJ software (USA). $\beta$-actin was the internal control for quantitative analysis of the relative expression levels of the proteins.

\section{Real-Time Polymerase Chain Reaction (RT-PCR)}

Rat brain tissue or microglia were treated with Trizol; the tissues were separated thoroughly with a homogenizer, and the mRNA was extracted using chloroform and isoamyl alcohol. The RNA sediment was dissolved in DEPC water (Takara, Shanghai, China), quantified using a spectrophotometer, and reverse transcribed into cDNA by using Prime Script reagent kits (Takara, Shanghai, China). PCR was performed using SYBR Green PCR kits (Takara, Shanghai, China) by a Step One Real-Time PCR System. Relative RNA Expression was normalized to $\beta$-actin and calculated. ${ }^{18}$ The primers used in the study are listed as follows: $\beta$-actin (5'-TTCGCCATGGATGACGATATC-3' and 5'- TAGGAGTCCTTCTGACCCATAC-3'), Cox-2 (5'TTCCAGTATCAGAACCGCATTGCC-3' and 5'-CCGTG TTCAAGGAGGATGGAGTTG-3'), IL- $\beta$ (5'-ATCTCAC AGCAGCATCTCGACAAG-3' and 5'-CACACTAGCAGG TCGTCATCATCC-3'), P2X7R (5'-CCACTCTGCTGCC TTGTCGTTAC- $3^{\prime}$ and $5^{\prime}$-CTGGTATGCGGTTAGATGC GATGG-3'), and TNF- $\alpha$ (5'-GCATGATCCGAGATGTGG AACTGG-3').

\section{Statistical Analysis}

All data were analyzed using GraphPad Prism 8. The results are expressed as mean $\pm \mathrm{SD}$. Comparisons between groups were performed using one-way analysis of variance and $t$-test. Pearson correlation analysis was used for twovariable correlation analysis. The $p<0.05$ was considered to be statistically significant. All experiments were repeated at least three times. 


\section{Results}

\section{Astaxanthin Alleviates Cognitive Dysfunction in SE Rats}

As shown in Figure 1A the weight of rats in SE group is lighter than the AST treated group $(* * p<0.01)$, and the seizure latency of $\mathrm{SE}+\mathrm{AST}$ group was longer than $\mathrm{SE}$ group in Figure 1B $\left({ }^{\#} p<0.01\right)$, which indicated that seizure frequency was decreased in AST treated group. And there was no statistically significant of survival rate between SE and AST treated group in Figure 1C. The Morris water maze is mainly used to test the memory and learning ability of experimental animals for spatial position and sense of direction. ${ }^{22}$ Figure $2 \mathrm{~A}$ shows that in the SE group, the escape latency of rats was significantly longer than that in the control group and that AST treatment significantly shortened the escape latency of SE rats in the hidden platform test. The result of the spatial probe test in Figure 2B shows that SE rats crossed the platform fewer times than the control group $(* * p<0.01)$ and AST-treated rats demonstrated an increase in the number of times they crossed the platform ${ }^{(\# \# p<0.01}$ vs $\mathrm{SE}$ ). Figure $2 \mathrm{C}$ shows that $\mathrm{SE}$ rats spent a lesser amount of time in the target quadrant than the control rats $(* * \mathrm{p}<0.01)$ and that AST-treated rats appeared to spend an extended amount of time in the target quadrant than the SE group ( ${ }^{\#} \mathrm{p}<0.01$ ). The results obtained from Figure 2 demonstrate that AST alleviated SE-induced cognitive dysfunction in rats.

\section{Astaxanthin Attenuates SE-Induced Hippocampal Neuron Damage}

The study demonstrated that AST treatment $(30 \mathrm{mg} / \mathrm{kg}$ ) alleviated SE-induced hippocampal damage in Nissl stain. Figure 3A reveals that hippocampal neural cell layer was disordered and the cellular structure was severely damaged in the SE group. This morphological change in the AST-treated group was effectively prevented. As shown in Figure 3B, the Nissl-stained hippocampal neurons decreased remarkably in the SE group in CA1 and CA3 regions of the hippocampus (* $p<0.05$ vs control), and the AST-treated group showed a lower loss of neuronal cells in the $\mathrm{CA} 1$ and $\mathrm{CA} 3$ regions of the hippocampus than the SE group ( ${ }^{\#} p<0.01$ vs.SE). These results suggested that AST had a protective effect on hippocampal neurons.
A

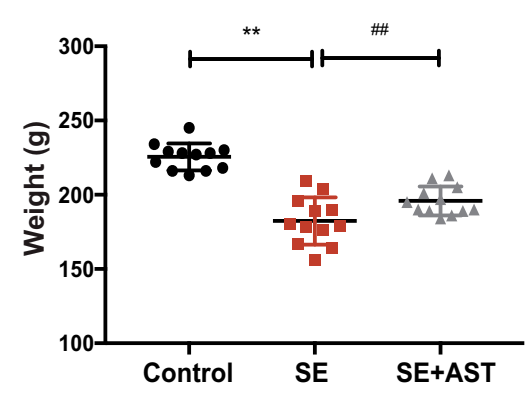

B

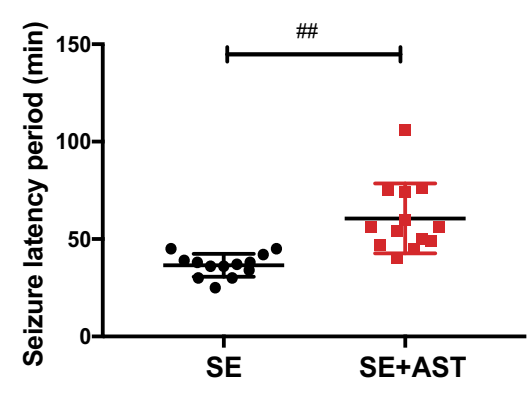

C

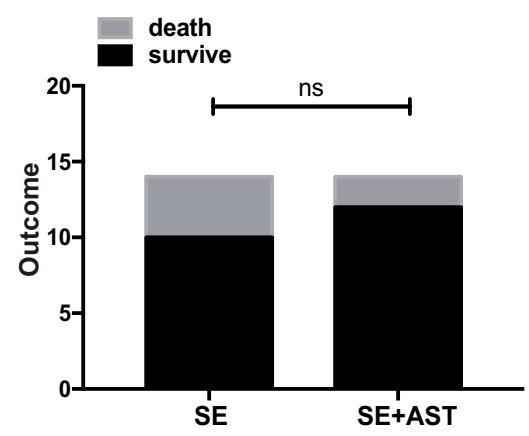

Figure I Data from the rat assessed in study. (A) The weight of rats in control $(n=12), S E(n=\mid 4)$, and SE+AST ( $n=\mid 4)$. (B) The seizure latency period to SE development in the SE and SE+AST group. (C) Rat survival after SE in SE and SE+AST group (**p $<0.01$ vs Control; ${ }^{* \#} p<0.01$ vs $\left.S E\right)$. Abbreviation: ns, not significant.

A

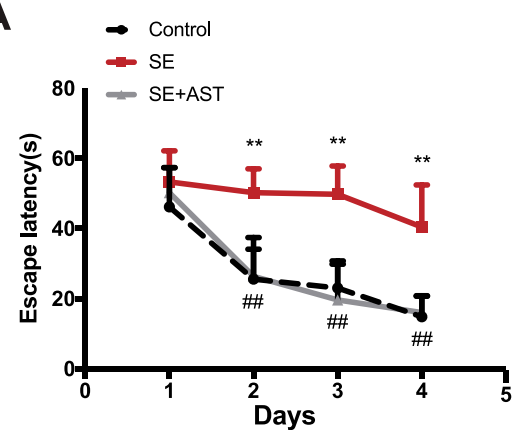

B

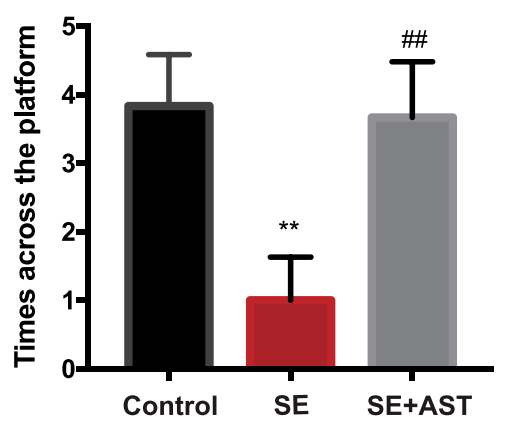

C

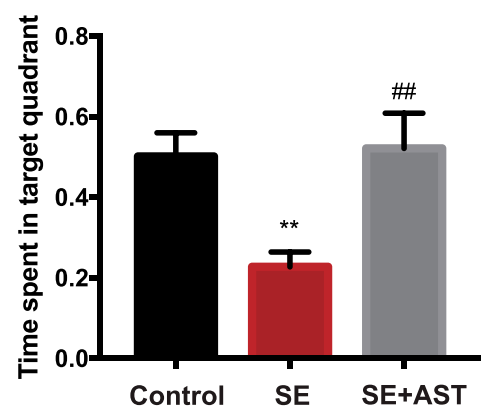

Figure 2 The Morris water maze test in rats. (A) The escape latency of rats. (B) The time across the platform. (C) The spent time of rats in target quadrant $(\mathrm{n}=\mathbf{8}$, $* * p<0.0$ I vs Control; ${ }^{\#} p<0.0$ I vs SE). 
A

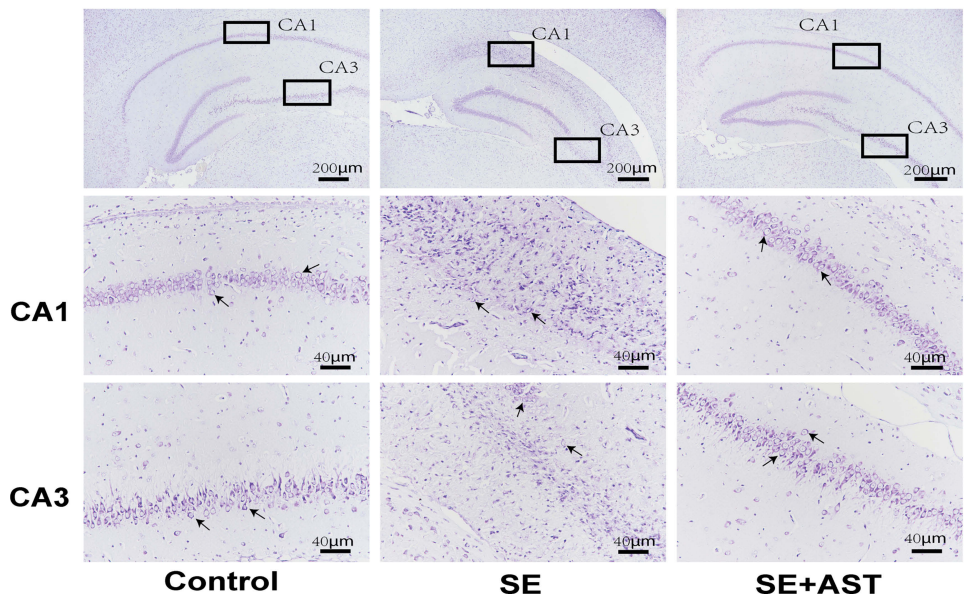

B

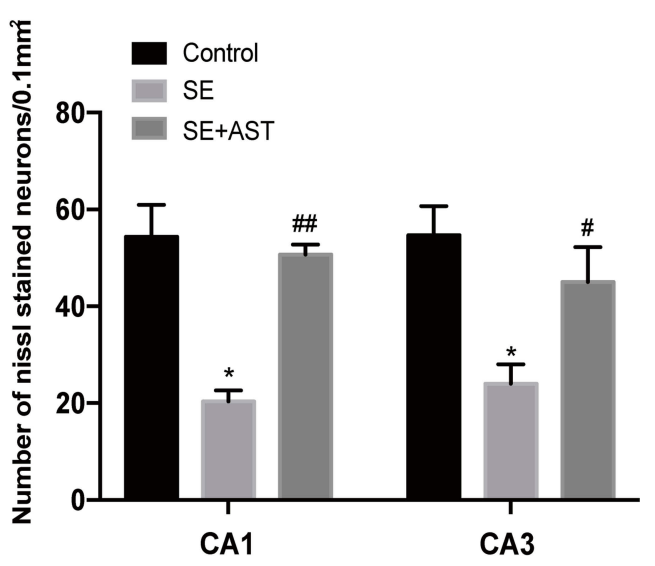

Figure 3 The Nissl stain of hippocampus in rats. (A) The Nissl stain of rat hippocampus. (B) The number of Nissl stained neurons. Left: control group. Middle: status epilepticus group. Right: astaxanthin (AST) treated group. The general morphology of the hippocampus in rat magnification 40x. The CAI and CA3 regions of hippocampus are marked in squares. $\mathrm{CAI}$ and CA3 region magnification $400 \times$. Positively stained cells are marked with black arrows. The cell density per square millimeter was measured by ImageJ software ( $n=4,{ }^{*} p<0.05$ vs Control; ${ }^{*} p<0.05$ vs SE; ${ }^{\#} p<0.01$ vs SE by one-way ANOVA).

\section{Astaxanthin Inhibits P2X7R-Related Neuroinflammation in SE Rats}

The inflammatory response during SE was accompanied by the activation, proliferation, and release of inflammatory cytokines in the microglia. ${ }^{12}$ Furthermore, P2X7R is a receptor on the surface of microglia, which mediates inflammation. ${ }^{9}$ Therefore, we further investigated the effect of AST on P2X7R-related inflammation in SE rats. Figure 4 and the associated results show that the number of microglia marked with Iba1 increased in the rat hippocampus than in the control group. AST treatment can effectively inhibit the microglial proliferation in CA1 and CA3 regions of the hippocampus. Additionally, our study detected the expression of P2X7R, TNF- $\alpha$, Cox- 2 , and IL- $1 \beta$ in rat brains by WB and real-time polymerase chain reaction (RT-PCR). As shown in Figure 5, the mRNA and proteins levels of P2X7R, TNF- $\alpha$, IL-1 $\beta$, and Cox-2 were significantly increased in the brains of the SE group rats ( $* p<0.05$ vs Control; ** $p<0.01$ vs Control). Furthermore, the expression of $\mathrm{P} 2 \mathrm{X} 7 \mathrm{R}$ is positive correlation with inflammatory cytokines IL- $1 \beta$ in the hippocampus of SE rats (Figure 5F). Compared to the SE group, AST treatment significantly reduced the expression levels of P2X7R, TNF- $\alpha$,
A
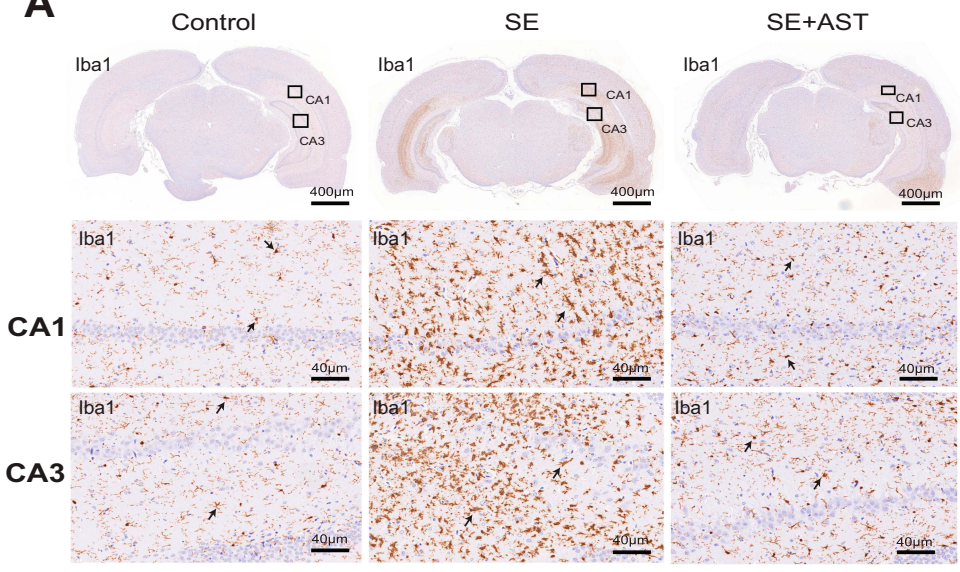

B

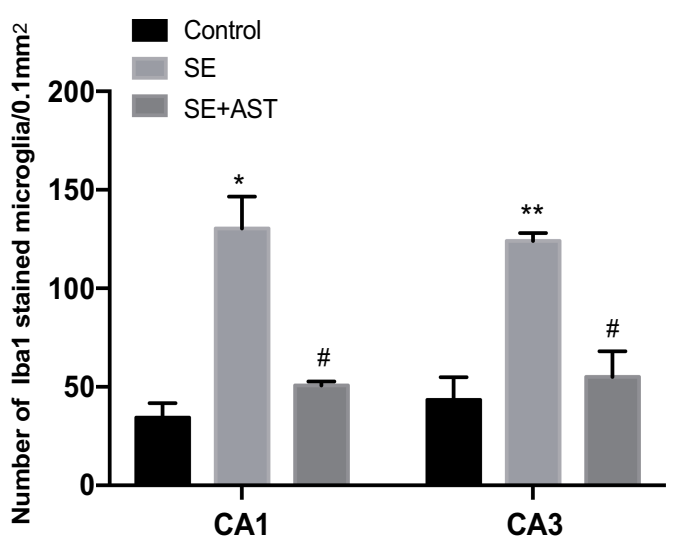

Figure 4 The immunohistochemistry stain in rats. (A) The immunohistochemistry stained of lbal in rat hippocampus. (B) The number of stained microglia. Left: control group. Middle: status epilepticus group. Right: astaxanthin (AST) treated group. The general morphology of the hippocampus in rat magnification $20 \times$. The CAI and CA3 regions of hippocampus are marked in squares. CAI and CA3 region magnification $400 \times$. Positively stained cells are marked with black arrows ( $n=4$, $* p<0.05$ vs Control; $*^{*} p<0.0$ I vs Control; ${ }^{*} p<0.05$ vs SE by one-way ANOVA). 
A

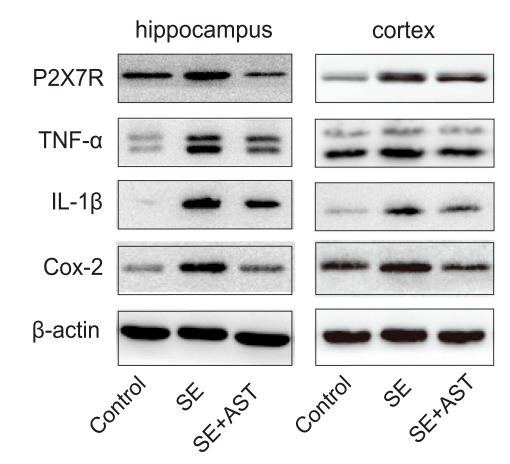

D

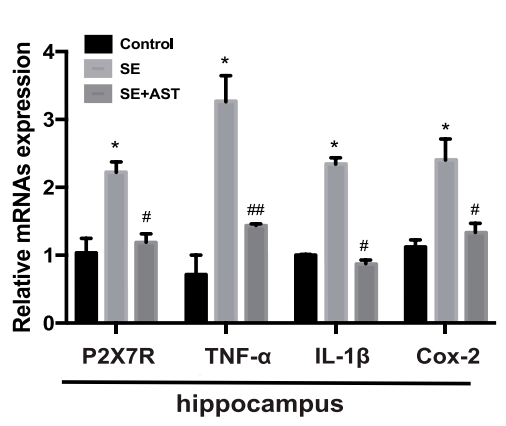

B

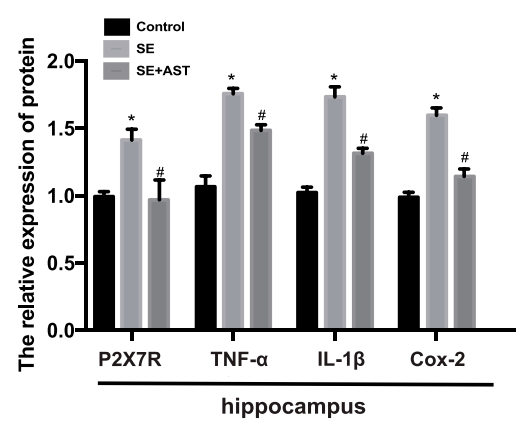

E

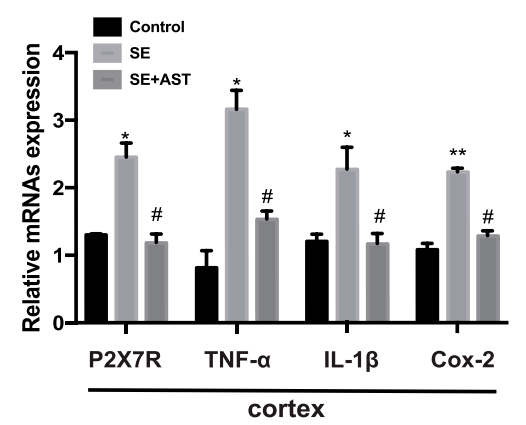

C

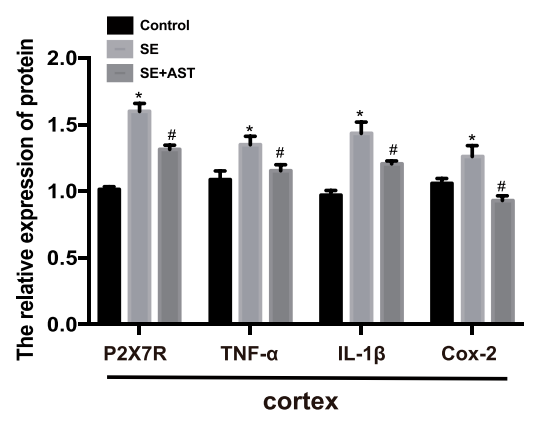

$\mathbf{F}$

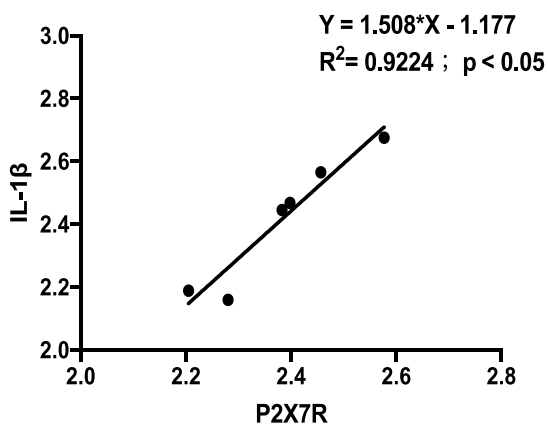

Figure 5 Effect of AST on P2X7R, IL-I $\beta$, Cox-2 and TNF- $\alpha$ expression in rats. (A) The protein P2X7R, TNF-a, IL-I $\beta$ and Cox-2 levels in cortex and hippocampus. (B) The relative protein levels of P2X7R, TNF- $\alpha$, IL-I $\beta$ and Cox-2 in hippocampus. (C) The relative protein levels of P2X7R, TNF- $\alpha$, IL-I $\beta$ and Cox-2 in cortex. (D) The relative mRNAs levels of P2X7R, TNF- $\alpha$, Cox-2 and IL-I $\beta$ in the hippocampus. (E) The relative mRNAs levels of P2X7R, TNF- $\alpha$, IL-I $\beta$ and Cox-2 in the cortex. (F) Pearson correlation analysis of mRNA expression of P2X7R and IL-I $\beta$ RNA in hippocampus of SE group. The relative expression of protein and mRNA were normalized to $\beta$-actin expression. Values are presented as the mean $\pm S D\left(n=5,{ }^{*} p<0.05,{ }^{* *} p<0.0\right.$ Ivs. Control; ${ }^{*}<0.05,{ }^{\#} p<0.0$ Ivs. SE).

IL-1 $\beta$ and Cox-2 both in the cortex and hippocampus of rats $\left({ }^{\#} p<0.05\right.$ vs SE; ${ }^{\#} p<0.01$ vs SE). The result demonstrated that neuroinflammation plays a vital role in SE-induced brain damage and that AST treatment $(30 \mathrm{mg} / \mathrm{kg})$ attenuated P2X7R-related neuroinflammation in the cortex and hippocampus of SE rats.

\section{Astaxanthin Downregulated the Expression of P2X7R and Inhibited Inflammation in LPS-Induced Microglia}

Since the potential mechanisms of AST inhibited neuroinflammation remain unclear, we further explored its effect on inflammation in vitro. And LPS-induced inflammation in rat microglia was used as an inflammation model to partly imitate neuroinflammation in SE rats. ${ }^{28}$ As shown in Figure 6, Cox-2 and IL-1 $\beta$ levels increased in microglia treated with different concentrations of LPS. At $10 \mu \mathrm{g} / \mathrm{mL}$, IL-1 $\beta$ and Cox-2 levels increased significantly compared to the control group $\left({ }^{*} p<0.05\right.$ vs control; ** $p<0.01$ vs control). The results in Figure $6 \mathrm{~B}-\mathrm{C}$ show that AST significantly suppressed Cox-2 and IL-1 $\beta$ expression at 50 $\mu \mathrm{M}$ in the LPS-stimulated microglia $(* p<0.05$ vs LPS; ** $p<0.01$ vs LPS). Therefore, we treated microglia with LPS $(10 \mu \mathrm{g} / \mathrm{mL})$ and AST $(50 \mu \mathrm{M})$ for $24 \mathrm{~h}$. The protein and mRNA levels of P2X7R, IL-1 $\beta$, and Cox-2 were measured using WB and RT-PCR to determine the effects of AST on inflammation. Figure 6D-F reveal that the P2X7R, Cox-2 and IL-1 $\beta$ levels in the LPS group increased significantly compared to the control group ( $* p<0.05$ vs Control group), suggesting microglial activation. And the AST reduced P2X7R, IL-1 $\beta$, and Cox-2 expression ${ }^{\#} p<0.05$ vs LPS group). Besides, microglia that were treated only with AST reduced the expression of P2X7R and IL-1b, but mildly elevated Cox $-2(* p<0.05$ vs control group). These results suggested that AST inhibited the P2X7R-related inflammation in vitro, which was consistent with the results obtained in vivo.

\section{Astaxanthin Regulated the ATP-P2X7R Signal in Microglia}

Figure $6 \mathrm{G}-\mathrm{H}$ show an increase in the extracellular ATP concentrations of microglia in the LPS group than in the control group $(* * p<0.01$ vs control). AST $(50 \mu \mathrm{M})$ treatment reduced the extracellular ATP concentration in LPS-induced 


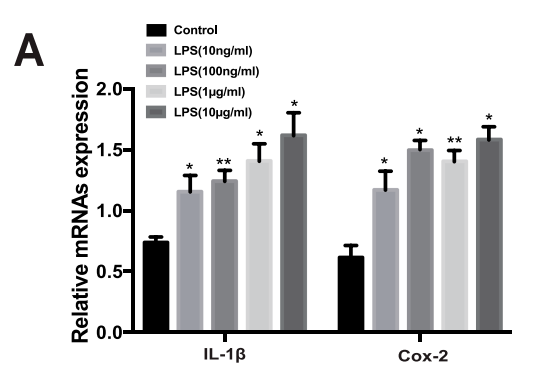

D

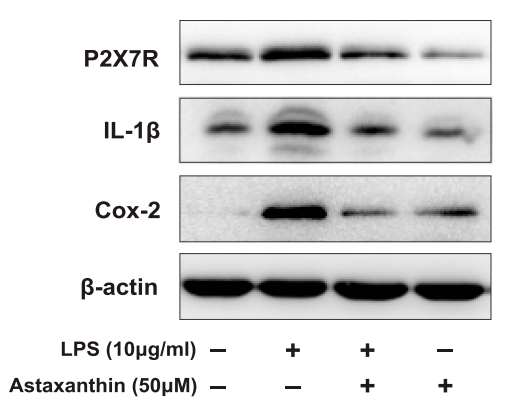

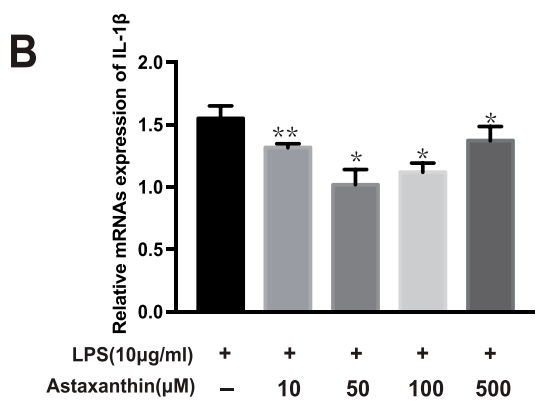

E

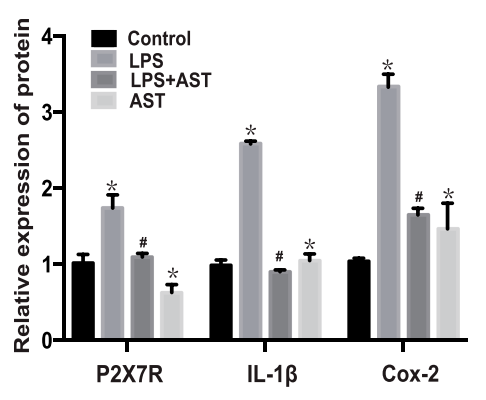

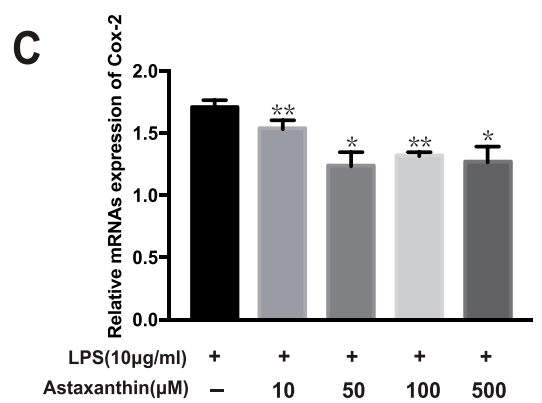

$\mathbf{F}$

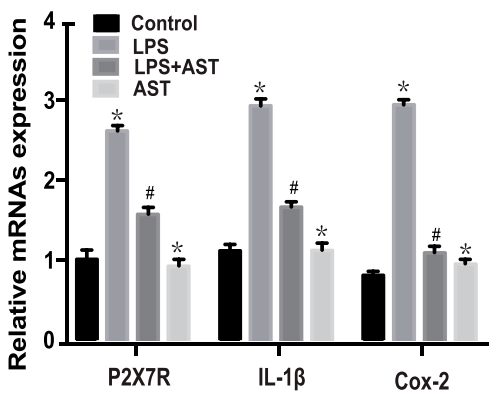

G

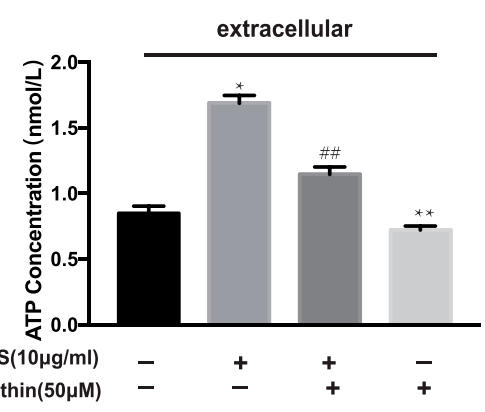

H

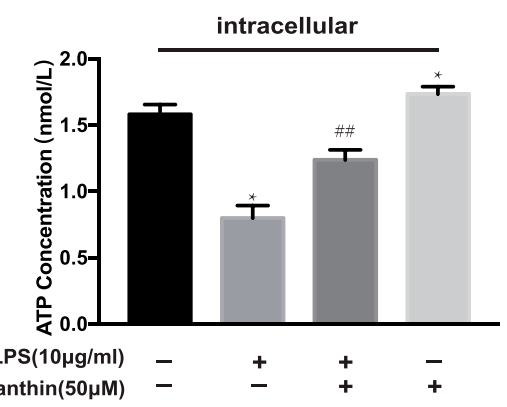

Figure 6 Effect of astaxanthin on P2X7R, IL-I $\beta$, Cox-2 and ATP expression in LPS-induced rat microglia. (A) The mRNA expression of IL-I $\beta$ and Cox-2 at different concentrations of LPS. (B, C) The mRNA expression of IL-I $\beta$ and Cox-2 treated with different concentrations of AST. (D) WB analysis for P2X7R, IL-I $\beta$ and Cox-2 protein levels in the rat microglia. (E) The expression levels of P2X7R, IL-I $\beta$ and Cox-2 proteins in microglia. (F) The mRNA expression of P2X7R, IL-I $\beta$ and Cox-2 were examined by RT-PCR. (G) The concentration of extracellular ATP $(\mathbf{H})$ The concentration of intracellular ATP. The relative expression of protein and mRNA were normalized to $\beta$ actin expression. The concentration of extracellular and intracellular ATP was measured by ELISA kit. Cells were treated with designed concentration of AST (50 $\mu$ M) and LPS $(10 \mu \mathrm{g} / \mathrm{mL})$ for $48 \mathrm{~h}$. Values are presented as the mean \pm SD $\left({ }^{*} p<0.05,{ }^{* *} p<0.01\right.$ vs Control group; ${ }^{*} p<0.05,{ }^{\#} \mathrm{p}<0.01$ vs LPS group).

microglia $\left({ }^{\#} p<0.01\right.$ vs LPS). In contrast, AST increased the intracellular ATP concentration in both the control and the LPS group ${ }^{\# \#} p<0.01$ vs LPS; $* * p<0.01$ vs Control). These results suggested that AST regulates the extracellular and intracellular ATP concentration conversely in microglia. As seen in Figure 7A and B, the expression of P2X7R at different ATP doses was distinct. P2X7R increased most significantly at ATP concentration of $1 \mathrm{mM}\left({ }^{*} p<0.05\right.$ vs Control) and BzATP treatment caused an increase of P2X7R higher than the ATP-induced ( $\& p<0.05$ vs ATP $0.1 \mathrm{mM}$ group). These results suggested that AST can decreased the expression of ATP directly and ATP can upregulated the expression of P2X7R, which indicted that AST regulated the ATP$\mathrm{P} 2 \mathrm{X} 7 \mathrm{R}$ in microglia.

\section{Astaxanthin Reduces Inflammation by Inhibiting the ATP-P2X7R Signal in \\ LPS-Stimulated Microglia}

As shown in Figure 7A and B, ATP treated increased the expression of $\mathrm{P} 2 \mathrm{X} 7 \mathrm{R}$ and inflammatory factors in microglia $\left({ }^{*} p<0.05\right.$ vs Control), which indicated that ATP-P2X7R signal can induced inflammation in microglia. And the result of Figure 7A and B showed that BzATP treated cause more intensive inflammation in microglia. BzATP is an agonist of $\mathrm{P} 2 \mathrm{X} 7 \mathrm{R}$, which has the stronger ability to activate P2X7R than ATP in vivo. Therefore, to further verify the effect of P2X7R on the inflammatory response, we used oxATP, an inhibitor of P2X7R, as a negative control for experiments. As shown in Figure 7C-E, similar to 
A

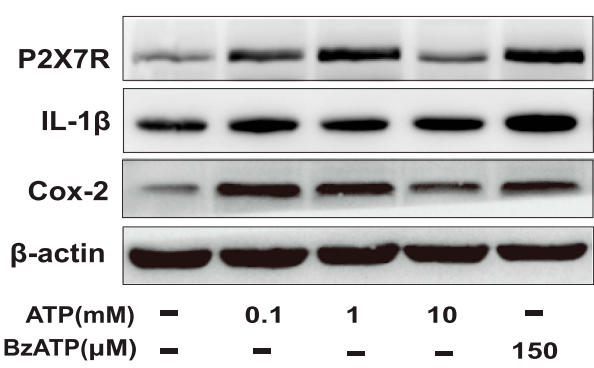

B

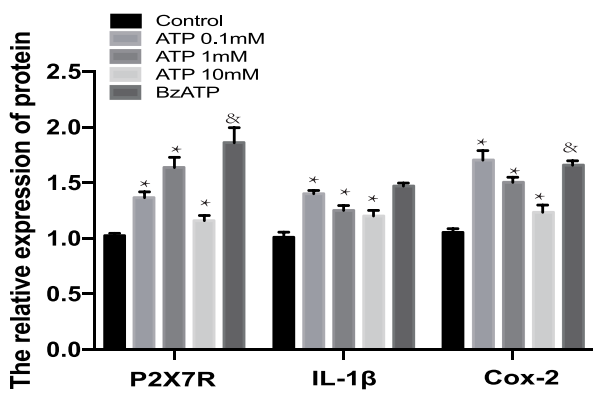

C

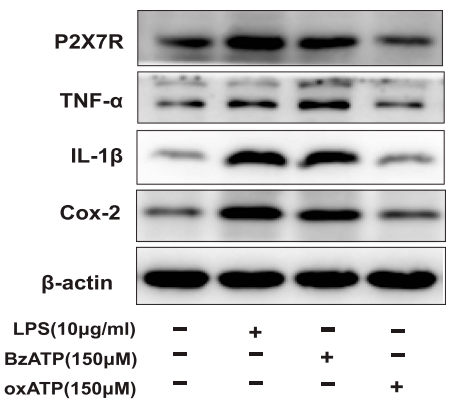

D

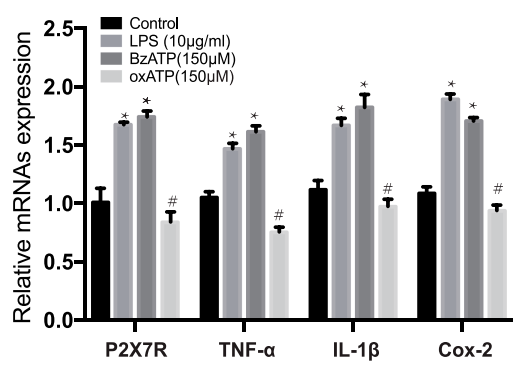

$\mathbf{E}$

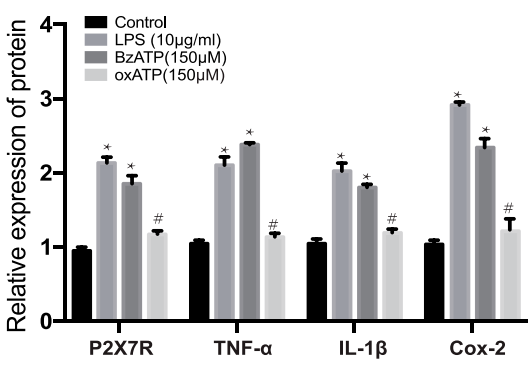

Figure 7 Effect of ATP on the P2X7R expression in microglia. (A) The protein expression of P2X7R, IL-I $\beta$ and Cox- 2 at different concentrations of ATP. (B) The relative protein of P2X7R, IL-I $\beta$ and Cox-2. (C) Western blot analysis for P2X7R, IL-I $\beta$ and Cox-2 protein levels in rat microglia. The expression level of P2X7R, TNF- $\alpha$, IL-I $\beta$ and Cox-2 proteins were normalized to $\beta$-actin expression. (D) The relative protein of P2X7R, IL-I $\beta$, TNF- $\alpha$ and Cox-2. (E) The relative mRNA expression of P2X7R, TNF- $\alpha$, IL-I $\beta$ and Cox- 2 were analyzed by RT-PCR in rat microglia. Cells were treated ATP $(0.1 \mathrm{mM}$, ImM and I0mM), LPS (I0 $\mu \mathrm{g} / \mathrm{mL})$, BzATP (I50 $\mu M)$, and oxATP (I50 $\mu$ M) for $48 \mathrm{~h}$ in each group. The relative expression of protein and mRNA were normalized to $\beta$-actin expression. Values are presented as the mean \pm SD ( ${ }^{*} p<0.05$ compared with the control group; ${ }^{\#}<0.05$ compared with the BzATP group; ${ }^{\&} p<0.05$ compared with the ATP ImM group).

the LPS-treated group, the expressions of P2X7R, TNF- $\alpha$, IL-1 $\beta$, and Cox-2 are significantly increased in BzATP treated group ( ${ }^{*} p<0.05$ vs Control). Conversely, oxATP treated downregulated the expression of $\mathrm{P} 2 \mathrm{X} 7 \mathrm{R}$ and P2X7R-related inflammation $\left({ }^{\#} p<0.05\right.$ vs BzATP). The results shown in Figure 7 indicate that ATP regulates the expression of P2X7R directly. Collectively, these studies found that AST attenuated LPS-induced inflammation by inhibiting the ATP-P2X7R signal.

\section{Discussion}

SE is both a neurological and systemic emergency that typically results in severe or fatal consequences. ${ }^{29}$ In the past few decades, a large number of studies have focused on the etiology, treatment, and prognosis of SE. ${ }^{30,31}$ A strong relationship between neuroinflammation and SE has been reported in previous studies. ${ }^{4,32}$ In both animal models and patients with SE, increasing evidence has shown that elevated inflammation in the brain during SE plays a decisive role in persistent seizures and long-term sequelae. ${ }^{33}$ In vivo evidence has shown that the site of inflammation contains high concentrations (hundreds of micromoles) of extracellular ATP, highlighting the vital role of extracellular ATP in inflammation. ${ }^{9}$ The ATP-gated purinergic P2X7R is an ion channel receptor that is primarily expressed on the membranes of immune cells such as microglia. It acts as a gatekeeper of neuroinflammation in the CNS, which subsequently mediates NLRP3 inflammasome activation and inflammatory cytokine release. ${ }^{10}$ A previous study observed an increase in P2X7R expression in SE rats. ${ }^{34}$ Furthermore, P2X7R antagonists are promising anti-inflammatory drug candidates for $\mathrm{SE}^{35}$ Similarly, this study demonstrated that treatment with anakinra, a pharmaceutical compound that interferes with the IL$1 \beta$ pathway, could effectively prevent cognitive dysfunction in SE rats. ${ }^{36}$ However, these drugs are far from application in SE owing to its unknown side effects. AST is the most powerful natural antioxidant found in nature. Numerous studies demonstrated the antioxidant, anti-neuroinflammatory, and neuroprotective effects of AST in animals with SE. ${ }^{17,37}$ Our previous study also showed the neuroprotective effect of AST on rats with SE. ${ }^{18}$ Furthermore, current studies have not reported any adverse effects of SE in human or animal experiments, along with the fact that it does not show side effects even at higher concentrations, which increases the potential application in 
clinical settings. ${ }^{12}$ However, certain anti-inflammatory mechanisms of AST require elucidation. Emerging evidence has shown that AST has a protective effect on mitochondria and inhibits the ATP efflux to maintain the energy supply to the cells. Additionally, it is a well-known fact that ATP is the agonist of P2X7R, which suggests that AST may inhibit the inflammation associated with the ATP-P2X7R signal. In addition, no previous research has reported the effect of AST on P2X7R. Therefore, we hypothesized that AST affects neuroinflammation by regulating the ATP-P2X7R signal in SE rats.

Inevitably, long-term seizures can lead to cognitive dysfunction, mental behavioral abnormalities, and decreased quality of life in patients with SE. The findings of our study show that AST plays a protective role in SE rats. With respect to experimental animal behavior, AST treatment extended the seizure latency and improved the cognitive function of SE rats in the Morris water maze test. Moreover, there was a reduction in the frequency of the seizures in the AST-treated group. In histomorphology, Nissl staining showed that ATS prevented hippocampus neurons from SE damage, and immunohistochemistry staining demonstrated that AST treatment significantly decreased the number of microglia in the hippocampus. With respect to the molecular biology, the inflammatory cytokines were significantly inhibited in the AST-treated group. These findings indicated that neuroinflammation after SE was the cause of hippocampal damage and cognitive dysfunction in SE rats. Treatment with AST effectively ameliorated the disease prognosis in SE rats. To clarify the neuro-protective mechanism of AST, we examined the differences in the expression of P2X7R between the SE and AST-treated rats. The data in Figure 5 show that AST downregulated the expression of P2X7R both in the hippocampus and cortex of SE rats. Furthermore, our result in Figure 5F consistent with the result that $\mathrm{P} 2 \mathrm{X} 7 \mathrm{R}$ is positively related to inflammation in SE rats as described in previous studies. ${ }^{9}$ Our study confirmed the critical role of P2X7R-mediated neuroinflammation in SE, which was reported in previous studies. ${ }^{9,27}$ Unlike them, we first discovered that AST attenuated neuroinflammation may through P2X7R signal. Therefore, we concluded that AST protected SE rats by inhibiting neuroinflammation, which was associated with P2X7R expression.

As a gateway to a neuroinflammatory reaction, $\mathrm{P} 2 \mathrm{X} 7 \mathrm{R}$ plays an important role in the development of neuroinflammation. Therefore, we further investigated the roles of AST on P2X7R. Microglia are the main immune cells in the brain that participate in the inflammatory reaction. ${ }^{38}$ It can be activated by various pathological stimuli of the central nervous system, including stroke, seizure, and trauma. The activated microglia can proliferate rapidly, secrete various inflammatory cytokines (TNF- $\alpha$, IL-1 $\beta$ and Cox-2), and lead to neuroinflammation. ${ }^{39}$ Furthermore, P2X7R is mainly expressed on the microglia. ${ }^{40}$ Therefore, we used LPS to induce microglia polarization to produce inflammation, ${ }^{41}$ which is similar to the inflammation in SE rats. This means that we use LPSinduced inflammation in microglia as a model of the inflammation in SE rats and further study the mechanism of AST on inflammatory response. Consistent with the results in SE rats, we found AST also downregulated P2X7R expression and inhibited the inflammation in LPSinduced microglia. Our findings confirmed the antiinflammatory effect of AST in both in vivo and in vitro experiments. It is widely accepted that AST has a protective effect on mitochondria, which is also known as the powerhouse of the cell. It has recently been reported that AST increased the intracellular ATP concentration in hepatic stellate cells. ${ }^{14}$ Furthermore, as an agonist of P2X7R, ATP plays an important role in the inflammatory reactions. ${ }^{10}$ Therefore, we detected the ATP concentration using ELISA both extracellularly and intracellularly. The results showed that AST decreased extracellular ATP levels in both the control and LPS- treated groups. Additionally, LPS treatment increased the level of extracellular ATP as reported in previous studies. ${ }^{10}$ Conversely, AST significantly decreased the intracellular ATP level in LPS-stimulated microglia. Furthermore, as seen in Figure 7A and B, ATP treated upregulated the expression of P2X7R, which indicated AST regulated LPS-induced inflammation through ATP-P2X7R signal in microglia. BzATP is an agonist of P2X7R, which has the stronger ability to activate P2X7R than ATP in vivo. Therefore, in order to further verify the effect of P2X7R on the inflammatory response, we used oxATP, an inhibitor of P2X7R, as a negative control for experiments. As shown in Figure 7, BzATP can directly upregulate the expression of P2X7R and P2X7R-mediated inflammation in microglia. Concurrently, oxATP decreased the expression of P2X7R and P2X7R-related inflammatory cytokines. In summary, this study revealed that AST downregulated the P2X7R expression by fixing the imbalance of intracellular and extracellular ATP concentrations, downregulated the expression of P2X7R and attenuated P2X7R-mediated inflammation in microglia. In addition, according to the calculation method in previous literature, microglia treated 
with AST of $50 \mathrm{uM}$ can reach the AST concentration in vivo, ${ }^{42}$ which indicated that AST inhibits inflammation both in vitro and vivo. AST is a potential new candidate for an anti-epileptic drug.

In general, our research has advantages and limitations. The advantage is that we first discovered AST can inhibit the inflammation by regulating the ATP-P2X7R signal and found that ATP can upregulate P2X7R expression directly. The limitations that need to be considered were follows: firstly, the inflammation in SE rats involved of many cells and LPS-induced inflammation only partially mimics the inflammation in SE rats; secondly, we did not inject $\mathrm{P} 2 \mathrm{X} 7 \mathrm{R}$ antagonists in vivo as controls to assessed the effect of astaxanthin; thirdly, the study lack of polyethylene glycol injection as a control group in rats. We hope to improve these limitations in future experiments. Further research is warranted to clearly understand the association between AST and neuroinflammation. Additionally, based on abundant preliminary research, AST may potentially become a new anti-epileptic drug in the future.

\section{Conclusion}

This study supports the protective effect of AST on SEinduced damage and reveals the molecular mechanism underlying AST in SE-induced inflammation. AST decreased the extracellular ATP concentration, thereby inhibiting P2X7R activation and upregulation, which causes the inhibition of the inflammatory signaling pathway and significantly suppresses the expression of inflammatory cytokine genes such as TNF- $\alpha$, Cox- 2 , and IL- $1 \beta$. Therefore, AST can attenuate neuroinflammation in SE by inhibiting the ATP-P2X7R signal.

\section{Ethics and Consent Statement}

All animal experiments were performed in compliance with institutional guideline and had been approved by the Ethics Committee of Jinshan Hospital of Fudan University (Certificate No. 20,141,601) and was operated according to the recommendations of the Chinese Academy of Medical Sciences Animal Experiment Guide.

\section{Data Sharing Statement}

All datasets generated in this study were included in the manuscript. The authors supported the original data in this manuscript will be available to any qualified researcher without undue reservation.

\section{Author Contributions}

All authors contributed to data analysis, drafting or revising the article, gave final approval of the version to be published, and agree to be accountable for all aspects of the work.

\section{Funding}

This research was funded by the National Natural Science Foundation of China, grant number 81971209.

\section{Disclosure}

The authors declare no conflict of interest.

\section{References}

1. Nelson SE, Varelas PN. Status epilepticus, refractory status epilepticus, and super-refractory status epilepticus. Continuum (Minneap Minn). 2018;24(6):1683-1707. doi:10.1212/CON.00000000000 00668

2. Lv RJ, Wang Q, Cui T, Zhu F, Shao XQ. Status epilepticus-related etiology, incidence and mortality: a meta-analysis. Epilepsy Res. 2017;136:12-17. doi:10.1016/j.eplepsyres.2017.07.006

3. Trinka E, Kalviainen R. 25 years of advances in the definition, classification and treatment of status epilepticus. Seizure. 2017;44:65-73. doi:10.1016/j.seizure.2016.11.001

4. Vezzani A, Dingledine R, Rossetti AO. Immunity and inflammation in status epilepticus and its sequelae: possibilities for therapeutic application. Expert Rev Neurother. 2015;15(9):1081-1092. doi:10.1586/14737175.2015.1079130

5. Vezzani A, Friedman A, Dingledine RJ. The role of inflammation in epileptogenesis. Neuropharmacology. 2013;69:16-24. doi:10.1016/j. neuropharm.2012.04.004

6. Rojas A, Jiang J, Ganesh T, et al. Cyclooxygenase-2 in epilepsy. Epilepsia. 2014;55(1):17-25. doi:10.1111/epi.12461

7. Christian CA. Persistent Protection against Pathology and Paroxysms by P2X7R Antagonism. Epilepsy Currents. 2018;18(1):42-44.

8. Janks L, Sharma CVR, Egan TM. A central role for P2X7 receptors in human microglia. $J$ Neuroinflammation. 2018;15(1). doi:10.1186/ s12974-018-1353-8

9. Beamer E, Fischer W, Engel T. The ATP-gated P2X7 receptor as a target for the treatment of drug-resistant epilepsy. Front Neurosci. 2017;11:21. doi:10.3389/fnins.2017.00021

10. Di Virgilio F, Dal Ben D, Sarti AC, Giuliani AL, Falzoni S. The P2X7 receptor in infection and inflammation. Immunity. 2017;47 (1):15-31. doi:10.1016/j.immuni.2017.06.020

11. Yu Y, Feng S, Wei S, et al. Extracellular ATP activates P2X7R-NF $-\kappa \mathrm{B}$ (p65) pathway to promote the maturation of bone marrowderived dendritic cells of mice. Cytokine. 2019;119:175-181. doi:10.1016/j.cyto.2019.03.019

12. Terrone G, Frigerio F, Balosso S, Ravizza T, Vezzani A. Inflammation and reactive oxygen species in status epilepticus: biomarkers and implications for therapy. Epilepsy Behav. 2019;101:106275. doi:10.1016/j.yebeh.2019.04.028

13. Kim SH, Kim H. Inhibitory effect of astaxanthin on oxidative stress-induced mitochondrial dysfunction-a mini-review. Nutrients. 2018;10(9):1137.

14. Bae M, Lee Y, Park YK, et al. Astaxanthin attenuates the increase in mitochondrial respiration during the activation of hepatic stellate cells. J Nutr Biochem. 2019;71:82-89. doi:10.1016/j.jnutbio.2019. 06.001 
15. Zuluaga M, Gueguen V, Letourneur D, Pavon-Djavid G. Astaxanthinantioxidant impact on excessive Reactive Oxygen Species generation induced by ischemia and reperfusion injury. Chem Biol Interact. 2018;279:145-158. doi:10.1016/j.cbi.2017.11.012

16. Hormozi M, Ghoreishi S, Baharvand P. Astaxanthin induces apoptosis and increases activity of antioxidant enzymes in LS-180 cells. Artif Cells Nanomed Biotechnol. 2019;47(1):891-895. doi:10.1080/ 21691401.2019.1580286

17. Lu Y, Wang X, Feng J, Xie T, Si P, Wang W. Neuroprotective effect of astaxanthin on newborn rats exposed to prenatal maternal seizures. Brain Res Bull. 2019;148:63-69. doi:10.1016/j.brainresbull.2019. 03.009

18. Deng X, Wang M, Hu S, et al. The neuroprotective effect of astaxanthin on pilocarpine-induced status epilepticus in rats. Front Cell Neurosci. 2019;13. doi:10.3389/fncel.2019.00123

19. Weissinger F, Wawra M, Fidzinski P, et al. Dentate gyrus autonomous ictal activity in the status epilepticus rat model of epilepsy. Brain Res. 2017;1658:1-10. doi:10.1016/j.brainres.2016.12.030

20. Meskinimood S, Rahimi N, Faghir-Ghanesefat H, Gholami M, Sharifzadeh M, Dehpour AR. Modulatory effect of opioid ligands on status epilepticus and the role of nitric oxide pathway. Epilepsy Behav. 2019;101(Pt A):106563. doi:10.1016/j.yebeh.2019.106563

21. Ishida S, Hamada K, Yagi K, Seino M. Comparing the anticonvulsive effects of dapsone on amygdala-kindled seizures and hippocampal-kindled seizures in rats. Acta Neurol Scand. 1992;85 (2):132-135. doi:10.1111/j.1600-0404.1992.tb04012.x

22. Zahra S, Lotfollah K, Farshad M, Ali MA, Ali P, Fatemeh Z. Nandrolone improve synaptic plasticity at the hippocampus CA1 area and spatial localization in the Morris water maze of male adolescent rats. Neurosci Res. 2019.

23. Wu W, Wu Y, Cheng G, Zhang C, Wang H, Li Y. A mouse model of hepatic ischemia-reperfusion injury demonstrates potentially reversible effects on hippocampal neurons and postoperative cognitive function. Med Sci Monit. 2019;25:1526-1536. doi:10.12659/ MSM.912658

24. Coronel-Oliveros CM, Pacheco-Calderon R. Prenatal exposure to ketamine in rats: implications on animal models of schizophrenia. Dev Psychobiol. 2018;60(1):30-42. doi:10.1002/dev.21586

25. Wesslau KP, Stein A, Kasper M, Barth K. P2X7 receptor indirectly regulates the JAM-A protein content via modulation of GSK-3 $\beta$. Int J Mol Sci. 2019;20(9):2298. doi:10.3390/ijms20092298

26. Xu Y, Xu Y, Wang Y, et al. Telmisartan prevention of LPS-induced microglia activation involves M2 microglia polarization via CaMKK $\beta$-dependent AMPK activation. Brain Behav Immun. 2015;50:298-313. doi:10.1016/j.bbi.2015.07.015

27. Jimenez-Pacheco A, Mesuret G, Sanz-Rodriguez A, et al. Increased neocortical expression of the $\mathrm{P} 2 \mathrm{X} 7$ receptor after status epilepticus and anticonvulsant effect of P2X7 receptor antagonist A-438079. Epilepsia. 2013;54(9):1551-1561. doi:10.1111/epi.12257
28. Liang Q, Yang R, Zhou Q, et al. MicroRNA-129-5p alleviates spinal cord injury in mice via suppressing the apoptosis and inflammatory response through HMGB1/TLR4/NF-kB pathway. Biosci Rep. 2020;40(3).

29. Hocker SE. Status Epilepticus. Continuum (Minneap Minn). 2015;21 (5 Neurocritical Care):1362-1383. doi:10.1212/CON.00000000000 00225

30. Betjemann JP, Lowenstein DH. Status epilepticus in adults. Lancet Neurol. 2015;14(6):615-624. doi:10.1016/S1474-4422(15)00042-3

31. Leitinger M, Holler Y, Kalss G, et al. Epidemiology-based mortality score in status epilepticus (EMSE). Neurocrit Care. 2015;22 (2):273-282. doi:10.1007/s12028-014-0080-y

32. Szklener S, Korchut A, Godek M, et al. Systemic inflammatory response syndrome in the course of status epilepticus: 7-year, two-center observational study. Epilepsy Res. 2017;137:53-55. doi:10.1016/j.eplepsyres.2017.09.003

33. Malter MP, Choi S, Fink GR. Cerebrospinal fluid findings in non-infectious status epilepticus. Epilepsy Res. 2018;140:61-65. doi:10.1016/j.eplepsyres.2017.12.008

34. Henshall DC, Engel T. P2X purinoceptors as a link between hyperexcitability and neuroinflammation in status epilepticus. Epilepsy Behav. 2015;49:8-12. doi:10.1016/j.yebeh.2015.02.031

35. Platania CBM, Giurdanella G, Di Paola L, et al. P2X7 receptor antagonism: implications in diabetic retinopathy. Biochem Pharmacol. 2017;138:130-139. doi:10.1016/j.bcp.2017.05.001

36. Dilena R, Mauri E, Aronica E, et al. Therapeutic effect of Anakinra in the relapsing chronic phase of febrile infection-related epilepsy syndrome. Epilepsia Open. 2019;4(2):344-350. doi:10.1002/epi4.12317

37. Otani H. Site-specific antioxidative therapy for prevention of atherosclerosis and cardiovascular disease. Oxid Med Cell Longev. 2013;2013:796891.

38. Leibowitz JA, Natarajan G, Zhou J, Carney PR, Ormerod BK. Sustained somatostatin gene expression reverses kindling-induced increases in the number of dividing type-1 neural stem cells in the hippocampi of behaviorally responsive rats. Epilepsy Res. 2019;150:78-94. doi:10.1016/j.eplepsyres.2019.01.005

39. Subhramanyam CS, Wang C, Hu Q, Dheen ST. Microglia-mediated neuroinflammation in neurodegenerative diseases. Seminars in Cell \& Developmental Biology. 2019;94:112-120.

40. Imura Y, Morizawa Y, Komatsu R, et al. Microglia release ATP by exocytosis. Glia. 2013;61(8):1320-1330. doi:10.1002/glia.22517

41. Li Y, Yang YY, Ren JL, Xu F, Chen FM, Li A. Exosomes secreted by stem cells from human exfoliated deciduous teeth contribute to functional recovery after traumatic brain injury by shifting microglia M1/ M2 polarization in rats. Stem Cell Res Ther. 2017;8(1):198. doi:10.1186/s13287-017-0648-5

42. Zuluaga Tamayo M, Choudat L, Aid-Launais R, et al. Astaxanthin complexes to attenuate muscle damage after in vivo femoral ischemia-reperfusion. Mar Drugs. 2019;17(6):354. doi:10.3390/ md17060354

\section{Publish your work in this journal}

Drug Design, Development and Therapy is an international, peerreviewed open-access journal that spans the spectrum of drug design and development through to clinical applications. Clinical outcomes, patient safety, and programs for the development and effective, safe, and sustained use of medicines are a feature of the journal, which has also been accepted for indexing on PubMed Central. The manuscript management system is completely online and includes a very quick and fair peer-review system, which is all easy to use. Visit http://www. dovepress.com/testimonials.php to read real quotes from published authors. 